\title{
Ontology Description using OWL to Support Semantic Web Applications
}

\author{
Rajiv Pandey \\ Asst. Professor \\ Amity University \\ Uttar Pradesh, Lucknow, India
}

\author{
Dr.Sanjay Dwivedi \\ Reader \& Head \\ Dr. BBA University \\ Lucknow, India
}

\begin{abstract}
In The World Wide Web (WWW) serves the human with vast amount of data and information.The usage pattern or user base has multiplied many folds since its origin. Despite the increasing importance gained by the WWW, to serve the human, it lacks the feature to serve meaningful information to machine this means that limited support for utilization of data and information is achieved. Thus the web needs to be made Semantic wherein different applications, agents, Web services and the web sites can exchange information to their full potential. This calls for representing the knowledge residing on WWW in a uniform manner understandable by both man and machine. Thus some taxonomy is needed to make representations of the web contents which can be machine readable and usable .This paper proposes to relate the need for Ontology and relate it to Web ontology language (OWL) and identify its position in making the Semantic Web. It is also felt that ontological support is needed for the semantic web in order to make the information ready for machine consumption. The ontological structure using the Web Ontology Language (OWL) which is used for modeling ontologies of context and for supporting Context reasoning is explored in this paper.The Web Ontology Language is designed for use by applications that need to process the content of information instead of just presenting information to humans. OWL facilitates greater machine interpretability of Web content
\end{abstract}

Keywords: Semantic Web, Ontology, OWL (Web ontology language).

\section{INTRODUCTION}

We The World Wide Web (WWW) has changed the way people interact/communicate with each Other and the way commerce is done. The WWW is serving human with information on Ecommerce, E-health and many more The constraint that still limits the utility of the Web is that it is supporting human interaction and most of the information on the web is meant for human consumption only, may it be viewing information making purchases etc. These activities are not particularly well supported by software agents or Web services. The WWW can be made more useful if the information available is made meaningful for the machine to interpret it thereby make the web Semantic. The Semantic web initiative of the World-Wide-Web consortium (W3C)[4] has been active for the past few years and efforts are on to make the current Web contents for machine interpretable.

This paper briefly evaluates the issues with the current web and the need for a common knowledge format which is suitable for both user and machine consumption.
This paper is organized in the following five sections: 1) introduction, 2) issues envisaging current web 3) Ontology support to the WWW 4) Semantic Web architecture and the ontology layer 5) ontology representation through Owl 6) Conclusion.

\section{ISSUES ENVISAGING CURRENT WEB}

The current web has grown manifolds since its origin this growth has not only been in the size of the number of users its serves [1] but also the volumes of pages that it stores. The WWW today supports E-commerce, E-health, E-education and E-governance activities to name a few, each of these need information in different formats to be understandable to the end users. It is also obvious that the data/objects (instances of classes) for Egovernance and E-health for a given geographical distribution may be common or identical .Thus the problem arises of data redundancy .Similarly two car dealers of a same parent supplier needs to maintain identical product database thus increasing the volume of data on the web and also leading to redundancy issues.

These problems can be addressed by storing the data at a common location and in a common format. Thus it is important to represent knowledge in a format which shall serve not only the user but also enhance the machine interpretation capabilities and based on the user need the results should be generated by querying a common database defined at a specified uniform resource indicator (URI). Thus the need for ontology arises which is discussed in the next paragraph.

\section{ONTOLOGY SUPPORT TO THE WWW}

Ontology is used to define a common vocabulary for particular domain. This vocabulary will be used to represent knowledge which can be shared by user and it will also be suitable for machine interpretability. Ontology as the term is used in the field of Knowledge representation and it is usually defined as "a representation of conceptualization" [2].An ontology defines the terms that may be used to describe or represent an area of knowledge [3].

Sharing common understanding of the structure of information or software agents is one of the most common purpose of ontologies [4,5] In context of the web, ontologies provide a shared understanding of a domain.

The ontology description shall help in the knowledge representation in more than one way and are listed as follows:

- It shall define a common structure of the information usable for both man and machine.

- It will enable reusability thus avoiding redundancy 
- It will separate the operational knowledge and the domain knowledge thus clearly specifying how knowledge is represented or stored and how it is fetched

- By defining classes, objects and their relationship it shall demonstrate the domain assumptions explicitly

- Enhance the analysis domain capabilities by proper inference of relation ships

Ontologies describe basic concepts in a domain, the classes, properties and also show the relationship between them thus it makes Knowledge reusable .It needs to describe the following concepts:

- Classes in many domains of interest as Windows with respect to house or relate it to computer operating system.

- The relation ship that may exists among the things

- The properties of the classes and their objects

Domain classification and description can give a clear meaning to the content stored. The hierarchy diagram (Fig-1) as shown below enables a more detailed interpretation of the meaning, their belongingness based on parent child relation ship and also the domain boundaries. The under mentioned hierarchy clearly infers that Dezire, Zen are a subclass of dealer2 which is a child of Car Dealer class. Thus the machine can interpret the meaning and generate results for query such as

- Dealer2 sells which cars.

- Dezire is sold by which dealer

It is thus clear that Ontologies can serve people, databases, Web services and also software agents who need to share information about a particular domain.

The diagram below is to a class hierarchy which is the basis for the ontology example to follow in the subsequent sections.

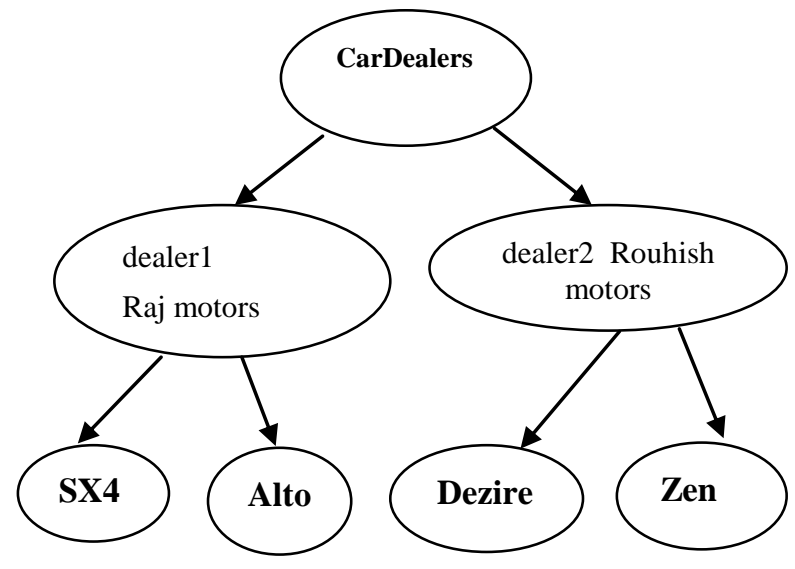

Figure1: Class Hierarchy to support machine interpretability.

\section{SEMANTIC WEB ARCHITECTURE AND THE ONTOLOGY LAYER}

To make the Web more appropriate for Machine consumption Tim Berners-Lee the creator of the Web had proposed the idea of semantic web in 2001 as "The Semantic Web will bring structure to the meaningful content of web pages, creating an environment where software agents roaming from page to page can readily carry out sophisticated tasks for users"[6] The architecture of semantic web suggested by Tim Berners-Lee has been the basis for research by many researchers today. The major issues encompassing the architecture are that there is no clear definition on the functionality of the various layers, the layers are also a combination of Functionality and technology and there is no precise definition to the various layers and there intended meanings, .

Before discussing a ontology syntax it is important that we have a look of The Sematic Web architecture and the purpose of ontology layer and understand the need of a onltology language to model Web Onltologies.

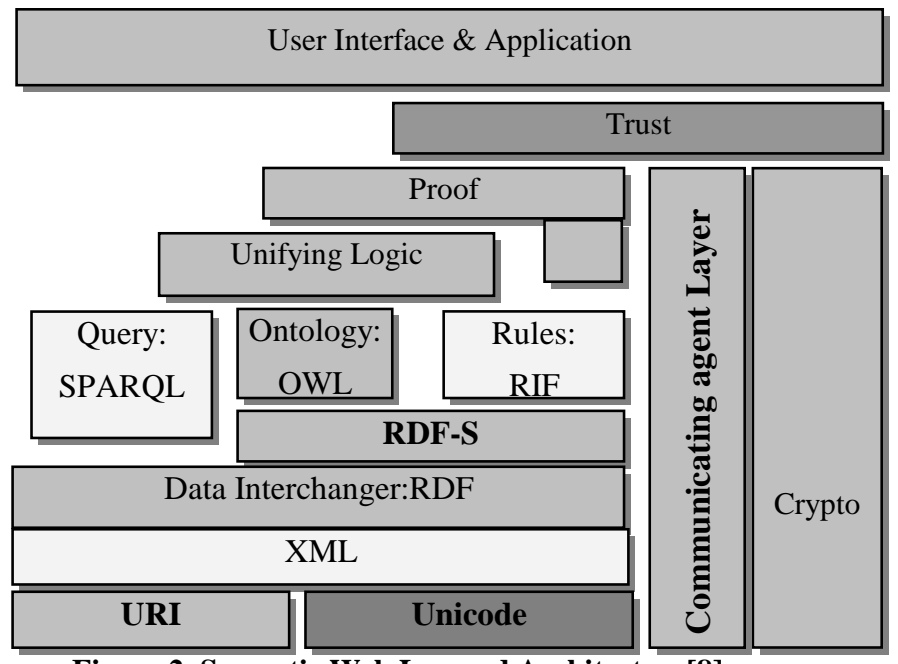

Figure 2. Semantic Web Layered Architecture[8]

The Semantic web architecture figure (2) [8] does show us that there are various layers described which support various functionalities and technologies to render machine understandability. The basic functionality of each layer is briefly explained so as to give a foundation to understand the need of ontology language.

The functionality of each layer with reference to the above layered architecture is thus as explained

1) Function of XML, XML schema and namespaces: XML, XML Schema and Namespaces, which are the components of layer 2, aim to be a baseline for structuring data on the web but without semantics. It is a mechanism used to describe data in a way that can be understood by the upper layers and can be interoperable.[9]

2) Function of RDF and RDF-S: The function of RDF and RDF-S(Resource Descriptive Framework-Schema)is to provide metadata to upper technologies placed on the layers on the top of layer 3 , in which that metadata can be exchanged and reused between these technologies or between these technologies and other applications.

3) Function of ontology layer: The main function of layer is the provision of semantics which produces a web of meaning [14]. Ontologies are helpful to clearly represent objects and also the relation ship between them it may be direct or inverse relationship. Using ontologies helps machines process meaning and facilitate sharing of information. 
4) Function of Rules layer : It is supposed to be used as a framework for making new inferences how these inferences should be expressed for the implementation of the Semantic Web.

5) Function of proof : This layer is incorporated to verify why the results generated by the agents should be believe $d$ or in other words the authenticity of the agent behavior is corroborated.

6) Function of Trust : The function of this layer is to provide a mechanism for trust and confidence between Information sources and information users(man or machine).

7) Function of Communicating agent layer(CAL) : CAL needs to perform the interoperability functions between various horizontal layers (Unicode to Proof) and the vertical layer crypto. This layer is a issue of research whether it should be a layer or a agent.

Thus we have understood for a search agent to retrieve semantics based information from the web it is important that the future web documents should be stored in a format where the ontology is clear and in order to create such documents we need a language which shall enable ontology definition. Thus the ontology layer in the figure(2) incorporates web Ontology Language(OWL).this paper shall briefly discuss OWL and a ontology example in the next section.

\section{ONTOLOGY REPRESENTATIONS THROUGH OWL}

\subsection{OWL: Web Ontology Language}

OWL $[3,15]$ is language by $\mathrm{W} 3 \mathrm{C}$ consortium OWL is a language for describing and creating instances of ontologies. The purpose of ontologies has been desribed in section 3 of this paper.Owl is a language which is based on XML/RDF syntax .The Owl ontology can normally be placed on Web Servers as normal web documents which can be referred by other ontologies and can be used by other applications, Web Services and software agents as the need be.

The under mentioned ontology example with certain modifications can be implemented using OWL editor Protégé, a free open-source editing framework developed by Stanford University.

\subsection{An OWL Ontology}

The ontology example below is with intent to give an understanding of the need of ontology and is not complete in itself and will need certain modification prior to implementations. The owl ontology described below has two primary parts

The first part shows the classes and the properties. The classes Car dealers and the cars, it also describes the "sells" and sold by" relation ship between the two.

The second part is a set of class instances and some imaginary domain boundaries.

The example below demonstrates a simple OWL construct. It is known that OWL helps us to define the various classes and subclasses. It also defines the relationship between the parent and child. This sample ontology definition is based on the support of RDF which further extends the XML tags. The example can be understood in brief with help of the Syntax and the details following them paragraph wise

$$
\begin{aligned}
& \text { <rdf : RDF } \\
& \text { xmlns= "http:// example.org /Cardealers\#" } \\
& \text { xmlns:exd="http://example.org/Cardealers\#" } \\
& \text { xmlns:owl= "http://www.w3.org/2002/07owl\#" } \\
& \text { xmlns:rdfs="http://www.w3.org/2000/01rdf-schema\#" } \\
& \text { xmlns:rdf="http://www.w3.org/1999/02/22-rdf-syntax-ns\#" } \\
& \text { xmlns:xsd= http://www.w3.org/2001/XMLSchema\#"> }
\end{aligned}
$$

The syntax above is defining the set of XML namespaces enclosed in the rdf:RDF opening tags. The namespaces included in the rdf;RDF tag are to specify the vocabulary for the other tags being used, $x m \ln s$ in this tag indicates that we are talking about the namespace where the definition for the other tags stored, the "xmlns $=\quad$ "http:// example.org /Cardealers\#"and "xmlns:exd=http://example.org/Cardealers\#" namespace asserts that this is the default namespace for this example and all unprefixed names refer to this ontology example, the subsequent statements of owl,rdfs,rdf,xml signify that the definition for these corresponding tags to be derived as stated by the respective namespaces. OWL constructs are XML based thus other namespaces specified in the RDF tag define the vocabulary for the same.

<owl:Ontology rdf:about = "http://example.org/Cradealers">

An ontology example based on cars and dealers.

$</$ rdfs:comments $>$

$<$ rdfs:label $>$ An Example Ontology</rdfs:label $>$

$</ o w l: O n t o l o g y>$

The above syntax block defines the metadata for the example that is being discussed it also encloses the comment "An ontology example based on cars and dealers." Which gives a human understandability aspect to the code and does not any way service machine purpose.

$<$ owl:Class rdf:ID= "CardealerNumber" $/>$

$<$ owl:Class rdf:ID $=$ "Cars" $/>$

This syntax defines the class definition of our example saying that there are two classes "Cardealers" and "Cars".

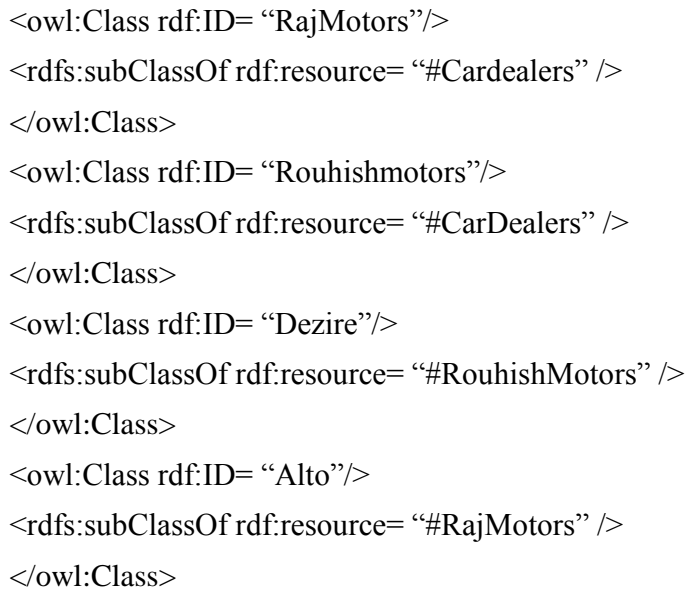




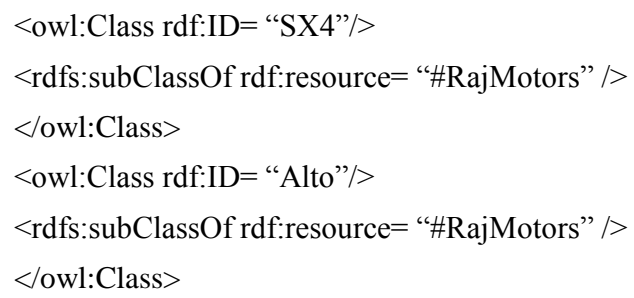

This part of the example establishes a class hierarchy of the CarDealers class explaining that RajMotors and Rouhish Motors are a sub class of Cardealers.It also explains that "SX4" and "Alto" are the subclasses of "RajMotors" Class and desire ,Zen are the sub class of RouhishMotors thus the whole of class hierarchy is established showing the parent child relationship.

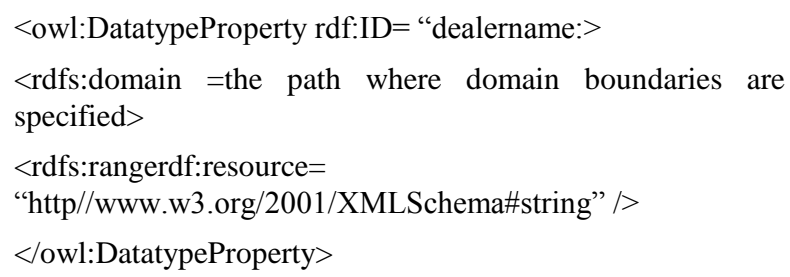

All objects of these classes should belong to a base class which is asserted by the domain aspect of the owl: Dataproperty, and the range aspect asserts that range value of the property must be of string type denoted by XmlSchema\#string

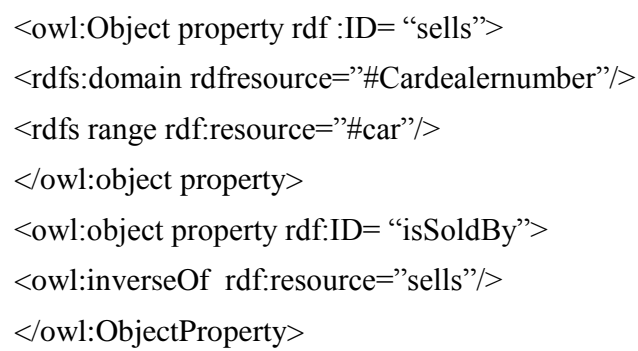

This block of the example explains the "sells" relationship and "isSoldBy" inverse relationship that will be exhibited by the instances of the classes shown by figure 2

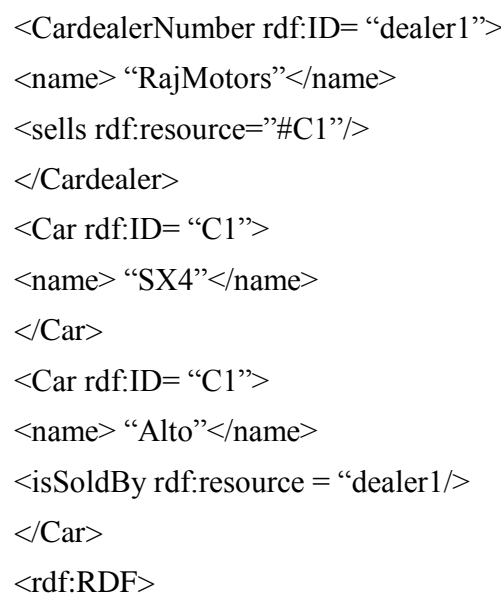

the above syntax block of the ontology decription shall now be abl;e to answer the questions as

- $\quad$ RajMotors sells which cars.

- Dezire is sold by which dealer
This shall be possible because the above syntax block establishes a Relation of "sells" and inverse relation of "isSoldBy" because the "sells"property will be able to relate the car dealer to the car and "isSoldBy" property shall relate a car to the cardealer as( Rajmotor "sells" Alto) and inversely (Alto "isSoldBy" RajMotors).

Thus it is clear that the ontology description shall give a semantic interpretation to the web documents and the machine can also interpret these documents and respond as the utility be.

\section{CONCLUSION}

Despite the need being felt to make today's web more machine interpretable and knowledge extractable there is a lot desired to make the semantic web a reality. This paper prompts the importance of the ontological definition of the Web contents which will not only keep the contents human usable but also add the feature of machine interpretability and after a meaningful interpretation the related contents can be integrated for a more optimal consumption. It is also observed that OWL will play a dominant role in the future for defining ontologies for supporting context reasoning and knowledge sharing. OWL shall thus play a significant role in turning the WWW to the Semantic Web and enable machine interpretability and human understandability

\section{REFERENCES}

[1] Net user stats can be referred at URL: http://internetworldststs.com/stats.htm last visited on 16-112010.

[2] T.R. Gruber, "towards principle for the design of ontologies used for knowledge sharing", International journal of human and computer studies, Vol.43, 1995

[3] OWL web ontology language Use case and requirements: W3C recommendations dated 10 February 2004

[4] M.A. Mussen,Dimensions of knowledge sharing and reuse.Computers and Biomedical research 25:435-467

[5] T.R. Gruber A translation approach to portable Ontology Specification. Knowledge Acquisition 5:199-220

[6] Tim Berners-Lee, James Hendler, Ora Lassila, "The Semantic Web", Scientific American, May 2001.

[7] Tim Berners-lee. Semantic Web Road Map. W3C Design Issues. URL http://www.w3. org/DesignIssues/Semantic.html, Oct. 1998.

[8] Rajiv pandey,Sanjay Dwivedi "Interoperability between semantic Web Layers: a Communicating Agent Approach" IJCA Dec 2010

[9] Haytam T.Al-feel,magdy Koutb,Hoda suoror. " Semantic Web on Scope: a new architectural model for the Semantic web", Journal of Computer Science 4(7):613-624,2008

[10] Ontology Definition Metamodel -OMG adopted Specification.ptc/06-10-11 dated October 2006

[11] http://www.w3.org/TR/2009/PR-owl2-primer20090922/OWL 2 Web Ontology Language Primer W3C Proposed Recommendation 22 September 2009 